\title{
The Elastic, Electronic and Thermodynamic Properties of a New Cd Based Full Heusler Compounds A Theoretical Investigation Using DFT Based FP-LMTO Approach
}

\author{
S. Aouimer ${ }^{a}$, M. Ameri $^{a, b}$, D. Bensaid ${ }^{a, c, *}$, N.E. Moulay ${ }^{a}$, A.Z. Bouyakoub $^{a}$,
} F.Z. BOUFAdi ${ }^{a}$, I. AMERI ${ }^{b}$ AND Y. Al-DOURI ${ }^{d, b}$

${ }^{a}$ Laboratory of Physical Chemistry of Advanced Materials, University of Djillali Liabes, BP 89, Sidi-Bel-Abbes 22000, Algeria

${ }^{b}$ Physics Department, Faculty of Science, University of Sidi-Bel-Abbes, 22000, Algeria ${ }^{c}$ Institute of Science, University Belhadj Bouchaib, BP 284, Ain-Temouchent, 46000, Algeria

${ }^{d}$ Nanotechnology and Catalysis Research Center (NANOCAT), University of Malaya, 50603 Kuala Lumpur, Malaysia

(Received October 21, 2018; revised version January 30, 2019; in final form February 20, 2019)

In this paper, we present the first principle calculation of the different properties of the full Heusler compounds $\mathrm{Cd}_{2} \mathrm{LaB}, \mathrm{Cd}_{2} \mathrm{PrB}$, and $\mathrm{Cd}_{2} \mathrm{CeB}$. Our calculations are based on density functional theory, using the full potential muffin-tin linear orbital method implemented in the LmtART code. For the determination of the exchange and correlation potential, we used the local density approximation and local spin density approximation. The elastic constants are also calculated. The total magnetic moment of $\mathrm{Cd}_{2} \mathrm{LaB}, \mathrm{Cd}_{2} \mathrm{PrB}$, and $\mathrm{Cd}_{2} \mathrm{CeB}$ using local spin density approximation are $3.334,3.482$, and $3.473 \mu_{\mathrm{B}}$ at ambient pressure, respectively. We also present the thermal effects using the quasi-harmonic Debye model, in which the vibrations of the network are taken into account. The effects of temperature and pressure on structural parameters, heat capacity, entropy, coefficient of thermal expansion, and the Debye temperatures are determined for unbalanced Gibbs functions.

DOI: 10.12693/APhysPolA.136.127

PACS/topics: FP-LMTO, full Heusler compounds, structural, magnetic moment, elastic properties, thermodynamic properties

\section{Introduction}

The Heusler alloys have attracted attention for its various applications, as well as their remarkable physical properties [1]. While there have been a number of works focused on the preparation and advanced characterization of structural and magnetic properties of these alloys, scientists are interested in these materials due to their extraordinary magnetic properties and industrial applications [2]. In addition to investigate thermal properties and to do a topological study of the electronic densities, another very rich objective is to investigate the derivation of the parameters that specify the equation of state (EOS) and related fundamental solid state properties, such as thermal expansion, melting temperature, and specific heat capacity. A study of the thermodynamic properties for these materials is well motivated by the understanding of the chemical bonds and the cohesion of material. Moreover, the elastic constants are related to thermal properties according to the Debye theory. Thermal properties can be unambiguously determined from the quasi-harmonic Debye model [3]. To undertake such

*corresponding author; e-mail: djizer@yahoo.fr investigation, ab initio calculations are performed with a state of the art electronic structure method, namely the full potential linear muffin-tin orbital (FP-LMTO) methodology, to study the structural, bonding, elastic, and thermodynamics properties of $\mathrm{Cd}_{2} \mathrm{LaB}, \mathrm{Cd}_{2} \mathrm{PrB}$, and $\mathrm{Cd}_{2} \mathrm{CeB}$ in its $L 2_{1}$ phase.

Knowledge about the elastic and thermodynamic properties is important for the material design and for other technical investigations. The elastic constants under pressure are very important to determine the response of the crystal to external forces, as characterized by the bulk and shear modulus and they obviously play an important role in determining the strength and hardness of the materials. Thermodynamic properties as a function of temperature and pressure may provide important information to understand the phase transitions and phase diagram. In this paper we present a first-principles study of structural, elastic, electronic, magnetic, thermodynamic properties and also the pressure effect on elastic constants of $\mathrm{Cd}_{2} \mathrm{LaB}, \mathrm{Cd}_{2} \mathrm{PrB}$ and $\mathrm{Cd}_{2} \mathrm{CeB}$. To the best of our knowledge, there are no experimental or theoretical works exploring the thermodynamic properties. This article is organized as follows: in Sect. 2, we briefly describe the computational techniques used in this work. We present the theoretical results and discussion of our work in Sect. 3. Finally we summarize the main conclusions of our work in Sect. 4. 


\section{Crystal structure and computational details}

The Heusler compounds [4] generally crystallize in the cubic $L 2_{1}$ structure (space group no. 225: $F m \overline{3} m) . \quad L 2_{1}$ structures are represented by the generic formula $\mathrm{X}_{2} \mathrm{YZ}$.

The full-Heusler structure consists of four penetrating face-centered cubic (fcc) sublattices with the $\mathrm{Z}$ and $\mathrm{Y}$ elements located at the $(0,0,0)$ and $(0.5,0.5,0.5)$, respectively, in the Wyckoff coordinates, while the X1 and X2 elements are at $(0.75,0.75,0.75)$ and $(0.25,0.25,0.25)$, respectively, resulting in two different rock-salt structures [X1Y] and [X2Z] as shown in Fig. 1 for the $\mathrm{Cd}_{2} \mathrm{LaB}$, $\mathrm{Cd}_{2} \mathrm{PrB}$, and $\mathrm{Cd}_{2} \mathrm{CeB}$ compounds.

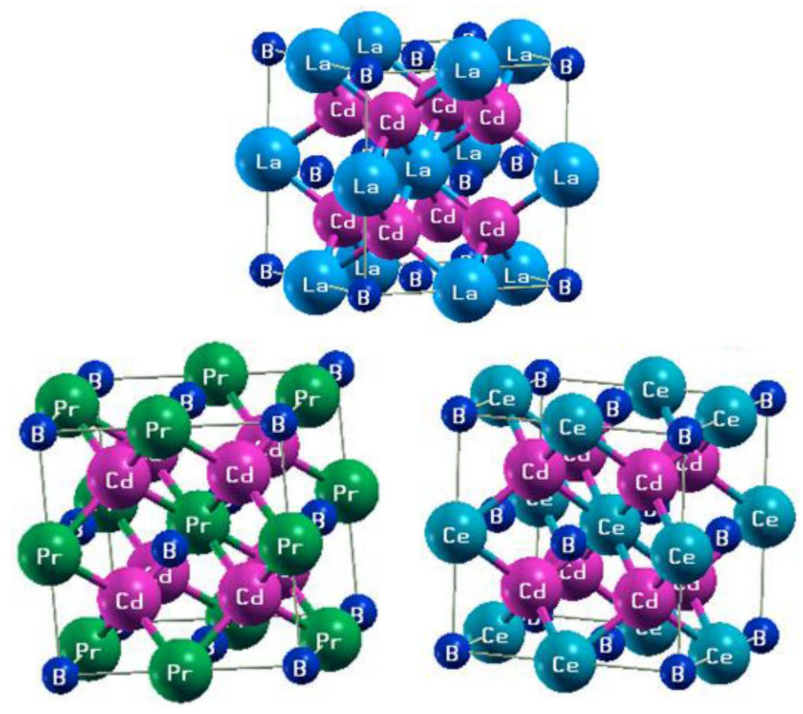

Fig. 1. The crystal structure of full Heusler compounds $\mathrm{X}_{2} \mathrm{YZ}$ for $\mathrm{Cd}_{2} \mathrm{LaB}, \mathrm{Cd}_{2} \mathrm{PrB}$ and $\mathrm{Cd}_{2} \mathrm{CeB}$.

The structural properties of the $\mathrm{Cd}_{2} \mathrm{LaB}, \mathrm{Cd}_{2} \mathrm{PrB}$ and $\mathrm{Cd}_{2} \mathrm{CeB}$ alloys are calculated using the FPLMTO method by performing local density approximation (LDA) and local spin density approximation (LSDA) [5, 6], with density functional theory (DFT). The multiple expansion of the crystal potential and the electron density within muffin-tin (MT) spheres were cut at $l_{\max }=10$. Non-spherical contributions to the charge density and potential within the MT spheres were considered up to $l_{\max }=6$. The cutoff parameter is $R_{\mathrm{mt}} K_{\max }=7$. In the interstitial region, the charge density and the potential are expanded as a Fourier series with wave vectors up to $G_{\max }=10 \mathrm{ua}^{-1}$. Using the energy eigenvalues and eigenvectors at these points, the density of states is determined by the tetrahedral integration method [7]. Table I contain the parameters of this calculation. the kinetic energy necessary to ensure the convergence ( $\left.E_{\text {cutoff }}\right)$, the number of plane waves used $\left(N_{P W}\right)$, and the radius of the sphere muffin-tin $\left(R_{M T}\right)$. We have chosen a Debye-like model as a simple way to consider the vibrational motion of the lattice.
TABLE I

The number of plane wave $N_{P W}$, energy cut-off $E_{\text {cut-off }}$ (in Ry) and the $R_{M T}$ (in a.u.) used in our calculations

\begin{tabular}{c|c|c|c}
\hline \hline Compounds & $R_{M T}$ & $N_{P W}$ & $E_{\text {cut }- \text { off }}$ \\
\hline \multirow{2}{*}{$\mathrm{Cd}_{2} \mathrm{LaB}$} & $\mathrm{Cd}: 2.796$ & 5064 & 72.76662 \\
& La:2.849 & & \\
& B: 2.849 & & \\
\hline \multirow{3}{*}{$\mathrm{Cd}_{2} \mathrm{PrB}$} & $\mathrm{Cd}: 2.733$ & 5064 & 67.27683 \\
& $\mathrm{Pr}: 2.733$ & & \\
& B: 2.733 & & \\
\hline \multirow{3}{*}{$\mathrm{Cd}_{2} \mathrm{CeB}$} & Cd: 2.764 & 5064 & 70.16094 \\
& Ce: 2.764 & & \\
& B: 2.764 & &
\end{tabular}

While retaining the simplicity of the Debye model, we have followed a quasi-harmonic approach, making the Debye temperature, $\theta(V)$, dependent upon the volume of the crystal. A reasonable alternative was to consider the isotropic approximation [11]. We apply here the quasiharmonic Debye model, implemented in the pseudo-code Gibbs [8-12]. Doing so, we could calculate the thermodynamic quantities at any temperature and pressure from the calculated $E-V$ data at $T=0$ and $P=0$. In addition, the elastic constants could also be calculated. Thermal properties could be determined from the quasiharmonic Debye model. To undertake these investigations, ab initio calculations were performed with the FPLMTO method in order to study the structural, bonding and thermodynamic properties of $\mathrm{Cd}_{2} \mathrm{LaB}, \mathrm{Cd}_{2} \mathrm{PrB}$, and $\mathrm{Cd}_{2} \mathrm{CeB}$.

\section{Results and discussions}

\subsection{Structural properties}

To determine the equilibrium lattice constant and to discover how the total energy varies with the cell volume, we performed structural optimizations on the $\mathrm{Cd}_{2} \mathrm{LaB}$, $\mathrm{Cd}_{2} \mathrm{PrB}$ and $\mathrm{Cd}_{2} \mathrm{CeB}$ full Heusler compounds.

The total energy dependence on the cell volume is fitted by the Murnaghan equation of state (EOS) given by [13]:

$$
E_{T}(V)=\frac{B_{0} V}{B_{0}^{\prime}}\left[\frac{\left(V_{0} / V\right)^{B_{0}^{\prime}}}{B_{0}-1}+1\right]+E_{0}-\frac{V_{0} B_{0}}{B_{0}^{\prime}-1},
$$

where $B_{0}$ is the bulk modulus, $B_{0}^{\prime}$ is the bulk modulus derivative, and $V_{0}$ is the equilibrium volume.

We summarized our results in Table II. There is no experimental data or theory available in the relevant literature which allows comparison with the specific results obtained on bulk coefficients. Figure 2 for $\mathrm{Cd}_{2} \mathrm{LaB}, \mathrm{Cd}_{2} \mathrm{PrB}$ and $\mathrm{Cd}_{2} \mathrm{CeB}$ display the total dependence energies on the cell size of the Heusler alloys. 

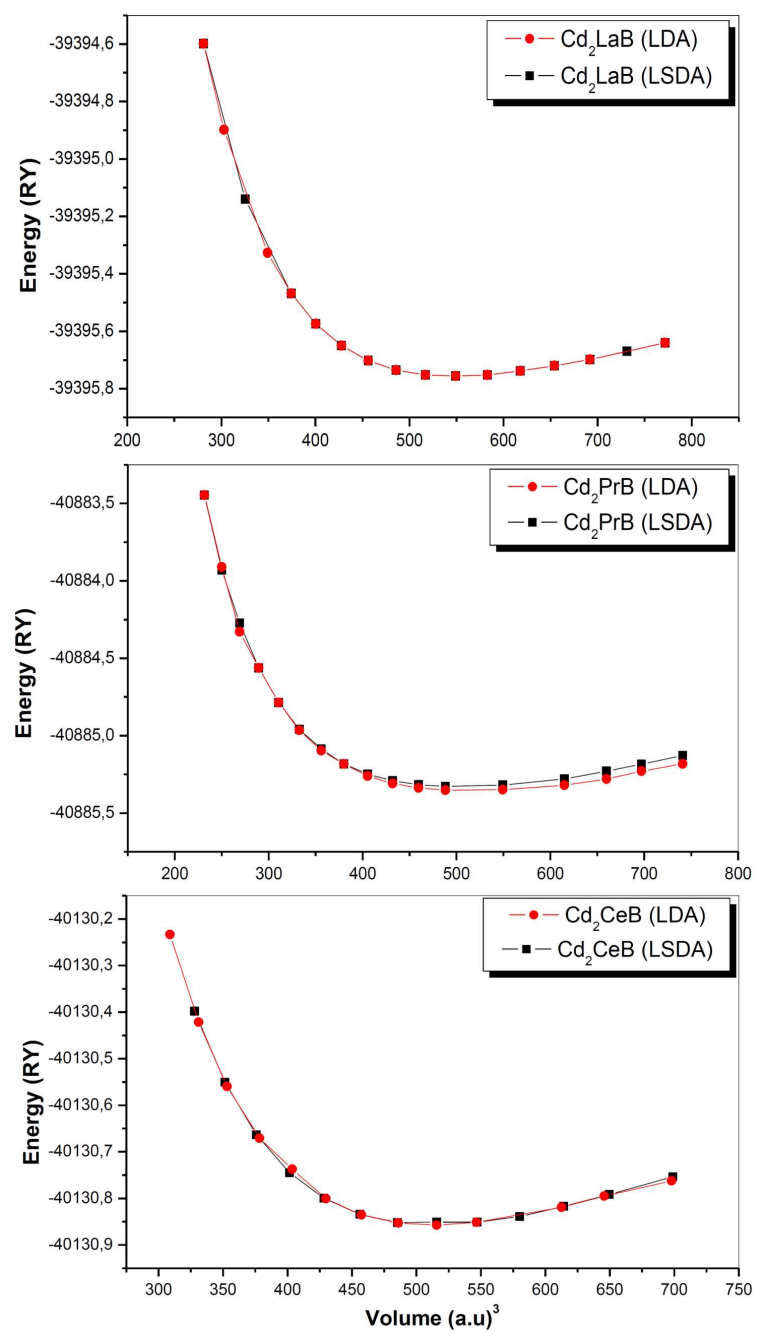

Fig. 2. Variation of the total energy versus the unit cell volume for the $\mathrm{Cd}_{2} \mathrm{LaB}, \mathrm{Cd}_{2} \mathrm{PrB}$, and $\mathrm{Cd}_{2} \mathrm{CeB}$ using LDA and LSDA.

TABLE II

The calculated values of the lattice parameter $a_{0}$ (in $\AA$ ), bulk modulus $B_{0}$ (in GPa) and its pressure derivative $B^{\prime}$ for $\mathrm{Cd}_{2} \mathrm{LaB}, \mathrm{Cd}_{2} \mathrm{PrB}$ and $\mathrm{Cd}_{2} \mathrm{CeB}$.

\begin{tabular}{c|c|c|c}
\hline \hline Compounds & $a_{0}[\AA]$ & $B_{0}[\mathrm{GPa}]$ & $B^{\prime}$ \\
\hline $\mathrm{Cd}_{2} \mathrm{LaB}$ & 6.90 & 75.705 & 3.748 \\
$\mathrm{Cd}_{2} \mathrm{PrB}$ & 6.68237 & 96.579 & 3.71257 \\
$\mathrm{Cd}_{2} \mathrm{CeB}$ & 6.757 & 79.476 & 4.18822
\end{tabular}

\subsection{Elastic properties}

The elastic constants of solids provide a link between the mechanical and the dynamic properties and provide important information about the nature of the action of the force in a solid. In particular, they provide information about the stability and stiffness of materials. Considering the cubic lattice, there are only three independent elastic constants, namely, $C_{11}, C_{12}$, and
$C_{44}$. By calculating the total energy as a function of strain, the elastic constants $\left(C_{11}, C_{12}\right.$, and $\left.C_{44}\right)$ are determined [14]. Based on the method developed by Mehl [15-17], by using the volume conserving tetragonal and orthorhombic strain, we can calculate the shear modulus $\left(C_{11}, C_{12}\right)$, and the elastic constant modulus $C_{44}$, respectively.

For calculating the difference of modulus of elasticity, $C_{11}-C_{12}$, a conserved volume orthorhombic stress tensor is given by the following expression

$$
\overleftrightarrow{\varepsilon}=\left[\begin{array}{ccc}
\delta & 0 & 0 \\
0 & \delta & 0 \\
0 & 0 & \frac{\delta^{2}}{1-\delta^{2}}
\end{array}\right],
$$

where $\delta$ is the applied constraint.

Applying this constraint affects the total energy

$$
E(\delta)=(-\delta)=E(0)+\left(C_{11}-C_{12}\right) V \delta^{2}+O\left[\delta^{4}\right]
$$

Here $E(0)$ is the energy of the system in the initial state (without constraint), $V$ is the volume of the unit cell. On the other hand, the compression modulus for an isotropic cubic crystal is written as a function of $C_{11}$ and $C_{12}$ as follows:

$$
B=\frac{1}{3}\left(C_{11}+2 C_{12}\right) .
$$

For the $C_{44}$ coefficient, a monoclinic stress tensor with a conserved volume is used with the following expression:

$$
\overleftrightarrow{\varepsilon}=\left[\begin{array}{ccc}
0 & \frac{\delta}{2} & 0 \\
\frac{\delta}{2} & 0 & 0 \\
0 & 0 & \frac{\delta^{2}}{4-\delta^{2}}
\end{array}\right] .
$$

The final form of this diagonal tensor is

$$
\overleftrightarrow{\varepsilon}=\left[\begin{array}{ccc}
0 & \frac{\delta}{2} & 0 \\
0 & -\frac{\delta}{2} & 0 \\
0 & 0 & \frac{\delta^{2}}{4-\delta^{2}}
\end{array}\right] .
$$

The total energy becomes

$$
E(\delta)=E(-\delta)=E(0)+\frac{1}{2} C_{44} V \delta^{2}+0\left[\delta^{4}\right] .
$$

By combining Eqs. (3.3) and (3.4), we can easily determine the two elastic constants $C_{11}$ and $C_{12}$, while the third elastic constant $C_{44}$ is deduced directly from Eq. (3.7).

Figures 3-5 represent the variation of total energy as a function of the stress for the compounds $\mathrm{Cd}_{2} \mathrm{LaB}$, $\mathrm{Cd}_{2} \mathrm{PrB}$, and $\mathrm{Cd}_{2} \mathrm{CeB}$ using the LDA approximation.

We can see that the value of bulk modulus $B=1 / 3$ $\left(C_{11}+2 C_{12}\right)$, calculated from the elastic constants is almost the same value as that obtained from the smoothing points $E_{\text {tot }}(V)$ using the Murnaghan state equation (EOS). This gives us a good estimate of the accuracy and precision of the elastic constants of $\mathrm{Cd}_{2} \mathrm{LaB}, \mathrm{Cd}_{2} \mathrm{PrB}$, and $\mathrm{Cd}_{2} \mathrm{CeB}$. Our calculated values for elastic constants LDA are presented in Table III. The traditional mechanical stability conditions of the elastic constants in cubic crystal are $C_{11}-C_{12}>0, C_{11}>0, C_{44}<0$, $C_{11}+2 C_{12}>0$ with the condition that $C_{11}$ constant must be lower than $C_{12}$ and $C_{11}<B<C_{12}[18,19]$. 

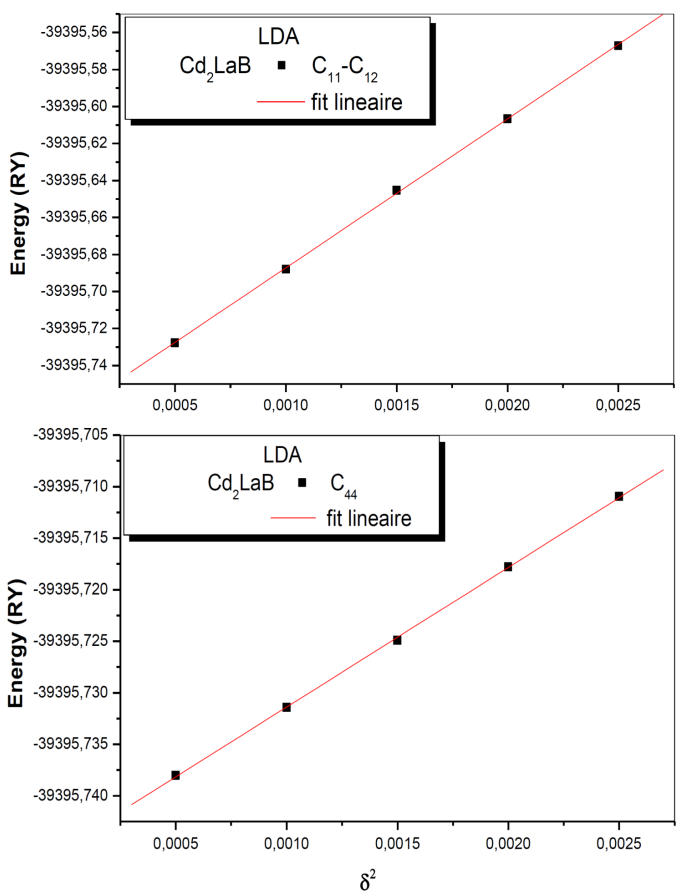

Fig. 3. Variation of the total energy as a function of the stress for the compound $\mathrm{Cd}_{2} \mathrm{LaB}$.

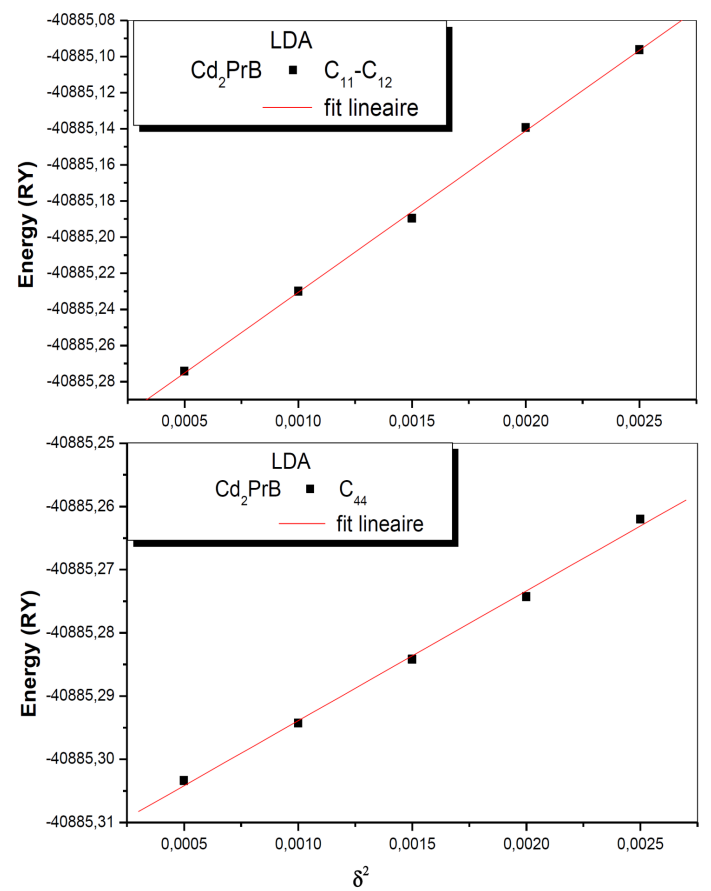

Fig. 4. As in Fig. 3, but for $\mathrm{Cd}_{2} \mathrm{PrB}$.

TABLE III

The elastic constants $C_{11}, C_{12}$ and $C_{44}$ (in GPa), the bulk modulus $B$ (in $\mathrm{GPa}$ ) for $\mathrm{Cd}_{2} \mathrm{LaB}, \mathrm{Cd}_{2} \mathrm{PrB}$ and $\mathrm{Cd}_{2} \mathrm{CeB}$.

\begin{tabular}{c|c|c|c|c}
\hline \hline Compounds & $C_{11}$ & $C_{12}$ & $C_{44}$ & $B[\mathrm{GPa}]$ \\
\hline $\mathrm{Cd}_{2} \mathrm{LaB}$ & 151.6709 & 38.56313 & 236.354 & 76.2657 \\
$\mathrm{Cd}_{2} \mathrm{PrB}$ & 176.8916 & 56.42266 & 312.695 & 96.5789 \\
$\mathrm{Cd}_{2} \mathrm{CeB}$ & 191.5456 & 23.44119 & 275.59 & 79.4759
\end{tabular}

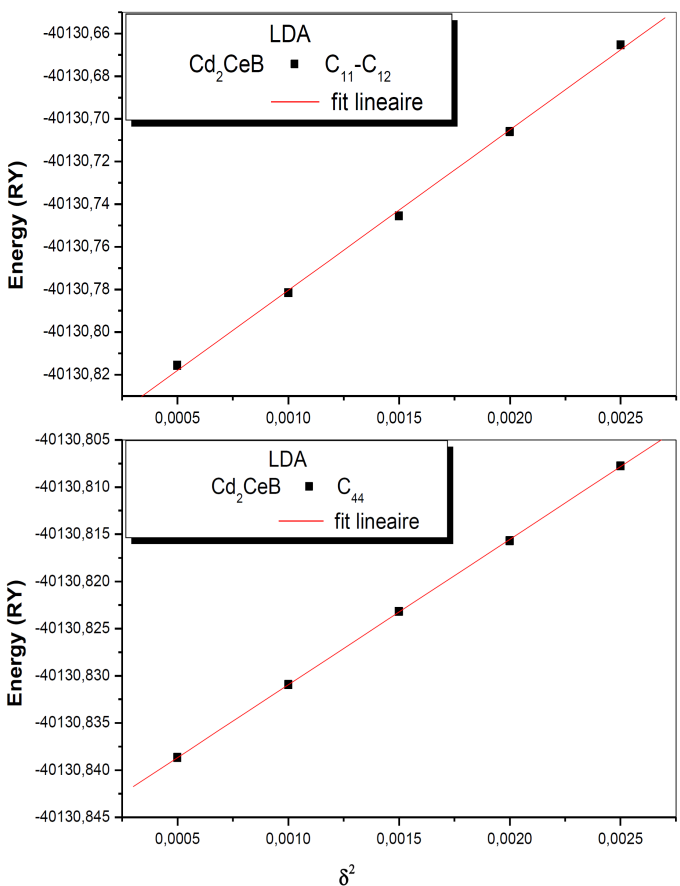

Fig. 5. As in Fig. 3, but for $\mathrm{Cd}_{2} \mathrm{CeB}$.

\subsection{Magnetic properties calculated in the LSDA}

Starting with the compound under investigation, all the information regarding the partial, total, and the previously calculated magnetic moments are summarized in Table IV. It is shown in Table IV that the calculated total magnetic moment is almost an integer value 3.334, 3.482, and $3.473 \mu_{\mathrm{B}}$ for $\mathrm{Cd}_{2} \mathrm{LaB}, \mathrm{Cd}_{2} \mathrm{PrB}$, and $\mathrm{Cd}_{2} \mathrm{CeB}$, respectively. It should be noted that $M_{\text {tot }}$ is the calculated total spin magnetic moment of the compound found by integration over the entire cell. Therefore, it is not just the combination of the moments at the Cd (2 times), Y (La, $\mathrm{Pr}, \mathrm{Ce})$, and $\mathrm{B}$ sites but respects also the moment of the interstitial between the sites.

TABLE IV

Total and partial magnetic moments in $\mu_{\mathrm{B}}$ (LSDA) for $\mathrm{Cd}_{2} \mathrm{LaB}, \mathrm{Cd}_{2} \mathrm{PrB}$ and $\mathrm{Cd}_{2} \mathrm{CeB}$

\begin{tabular}{c|c|c|c|c}
\hline \hline $\mathrm{X}_{2} \mathrm{YZ}$ & $M_{\text {tot }}$ & $M_{\mathrm{Cd}}$ & $M_{\mathrm{Y}}$ & $M_{\mathrm{B}}$ \\
\hline $\mathrm{Cd}_{2} \mathrm{LaB}$ & 3.334 & -0.157 & -0.360 & 0.118 \\
$\mathrm{Cd}_{2} \mathrm{PrB}$ & 3.482 & 0.256 & 2.092 & -0.380 \\
$\mathrm{Cd}_{2} \mathrm{CeB}$ & 3.473 & 0.501 & 0.904 & 0.424
\end{tabular}

\subsection{Electronic band structure}

We studied the band structure of the (a) $\mathrm{Cd}_{2} \mathrm{CeB}$, (b) $\mathrm{Cd}_{2} \mathrm{PrB}$, and (c) $\mathrm{Cd}_{2} \mathrm{LaB}$ compounds using the LSDA approximation.

The energy bands give the possible energies of an electron as a function of the wave vector. These bands are therefore represented in the reciprocal space, and for simplicity, only the directions between the points of high symmetries in the first Brillouin zone are treated. 
(a)

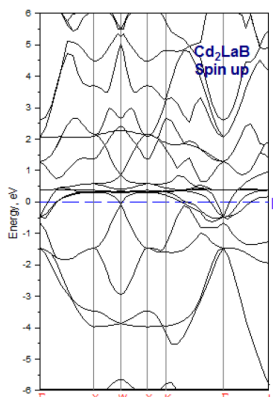

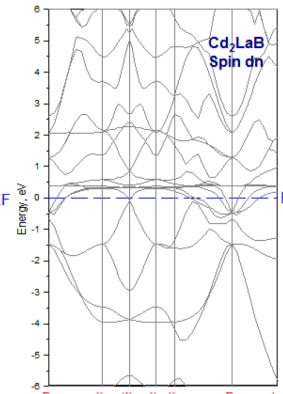

(b)

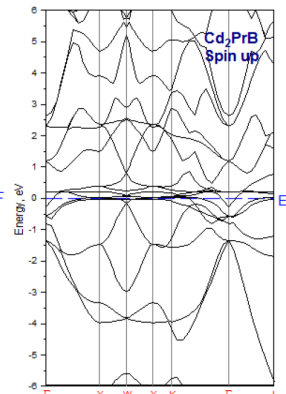

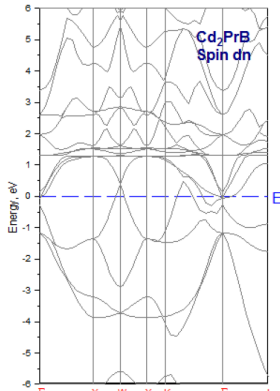

(c)

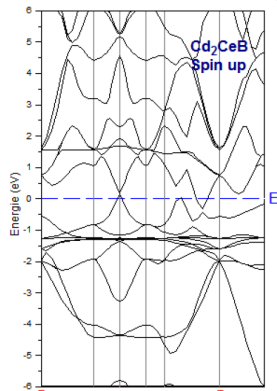

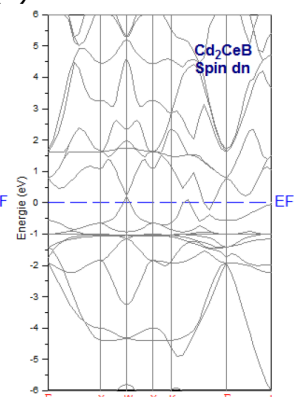

Fig. 6. The band structure for the spin up ( $\uparrow)$ and the spin down $(\downarrow)$ of (a) $\mathrm{Cd}_{2} \mathrm{CeB},(\mathrm{b}) \mathrm{Cd}_{2} \operatorname{PrB}$, and $(\mathrm{c}) \mathrm{Cd} \mathrm{L}_{2} \mathrm{LaB}$ alloys.

For semiconductors, the spectrum is characterized by the presence of three bands, the valence band BV, the band $E_{g}$ forbidden or the gap and the band BC of conduction, from the lowest energy towards the higher energies, respectively.

The energy gap is defined as the difference between the maximum of the valence band and the minimum of the conduction band. For the compounds $\mathrm{Cd}_{2} \mathrm{YB}(\mathrm{Y}=\mathrm{La}$, $\mathrm{Pr}$, and $\mathrm{Ce}$ ), we note that the minimum of the conduction band and the maximum of the valence band are located at the Fermi level of the first Brillouin zone. There is an overlap of band at this level, i.e. the electrons move freely between the valence band and the conduction band under ambient conditions.

Precisely at point $\Gamma$, the energy gap equals zero in the first Brillouin zone, so these compounds are metals for the majority and minority spin, as depicted in Fig. 6a-c.

\subsection{Thermodynamic properties}

We applied the quasi-harmonic Debye model [20] to obtain the thermodynamic properties of the $\mathrm{Cd}_{2} \mathrm{LaB}$, $\mathrm{Cd}_{2} \mathrm{PrB}$, and $\mathrm{Cd}_{2} \mathrm{CeB}$ compounds through the calculation of the $E-V$. The first step is to calculate the total energy versus the primitive cell volume $(E-V)$. The obtained results are then fitted with a numerical EOS in order to determine the structural parameters at $P=0 \mathrm{GPa}$ and $T=0 \mathrm{~K}$. The macroscopic properties are derived as a function of $P$ and $T$ from the standard thermodynamic relationship. The thermal properties are determined in the temperature range from 0.0 to $1400 \mathrm{~K}$ where the quasi-harmonic model remains fully valid. The pressure effect was studied in the 0 to $40 \mathrm{GPa}$ range. The variation of the volume with respect to the temperature at different pressures is shown in Fig. 7.

The volume is found to increase slightly with increasing temperature. The variation of the bulk modulus $B$ as a function of the temperature at a constant pressure is shown in Fig. 8.

The bulk modulus is found to decrease with increase of temperature at a constant pressure and also to increase with pressure at a constant temperature. In Fig. 9, it can be seen that the impact of the temperature variation on the Debye temperature is less than that of the pressure, and also that the decreasing trend is nearly linear for each pressure.
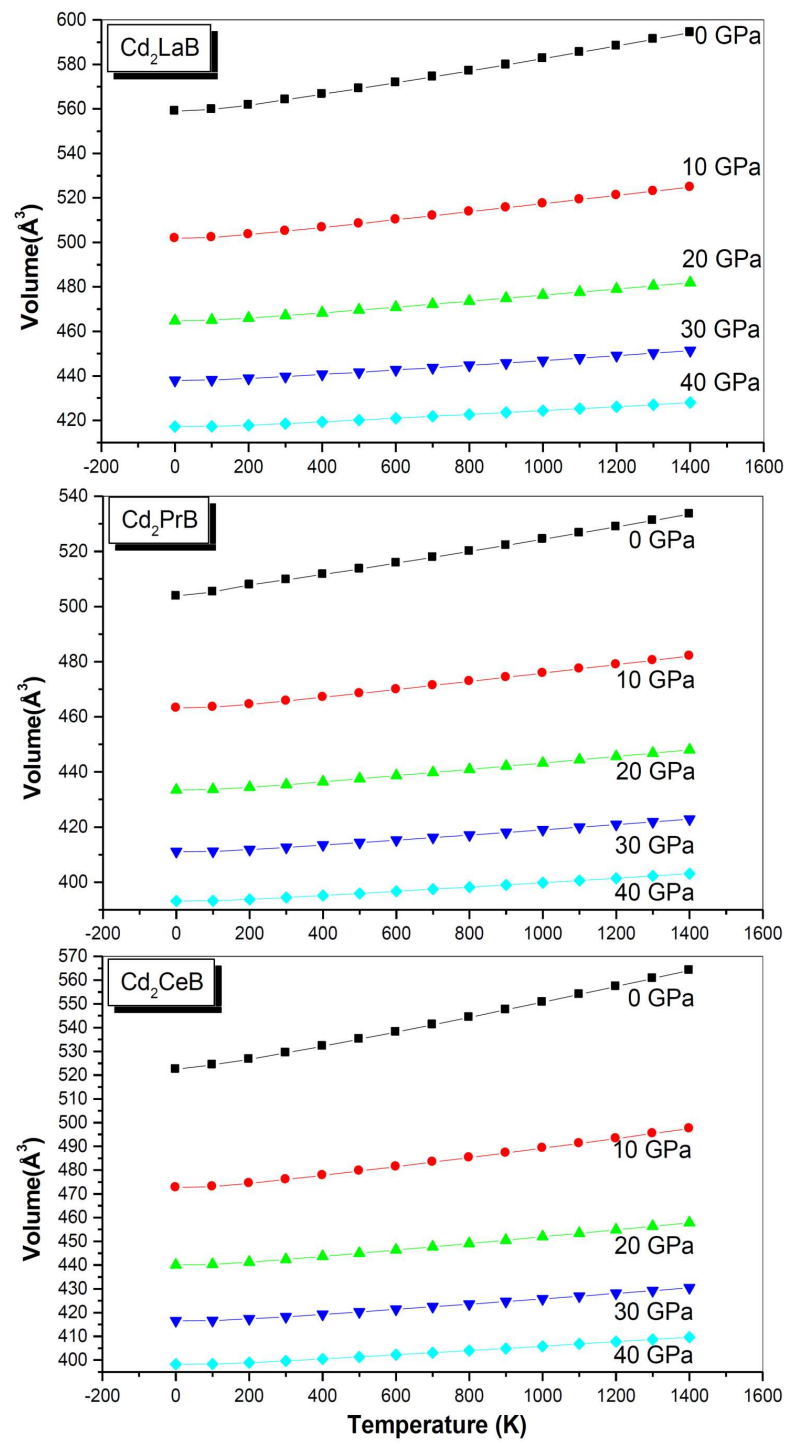

Fig. 7. The temperature effects on the lattice parameter of $\mathrm{Cd}_{2} \mathrm{LaB}, \mathrm{Cd}_{2} \mathrm{PrB}$, and $\mathrm{Cd}_{2} \mathrm{CeB}$ alloys. 

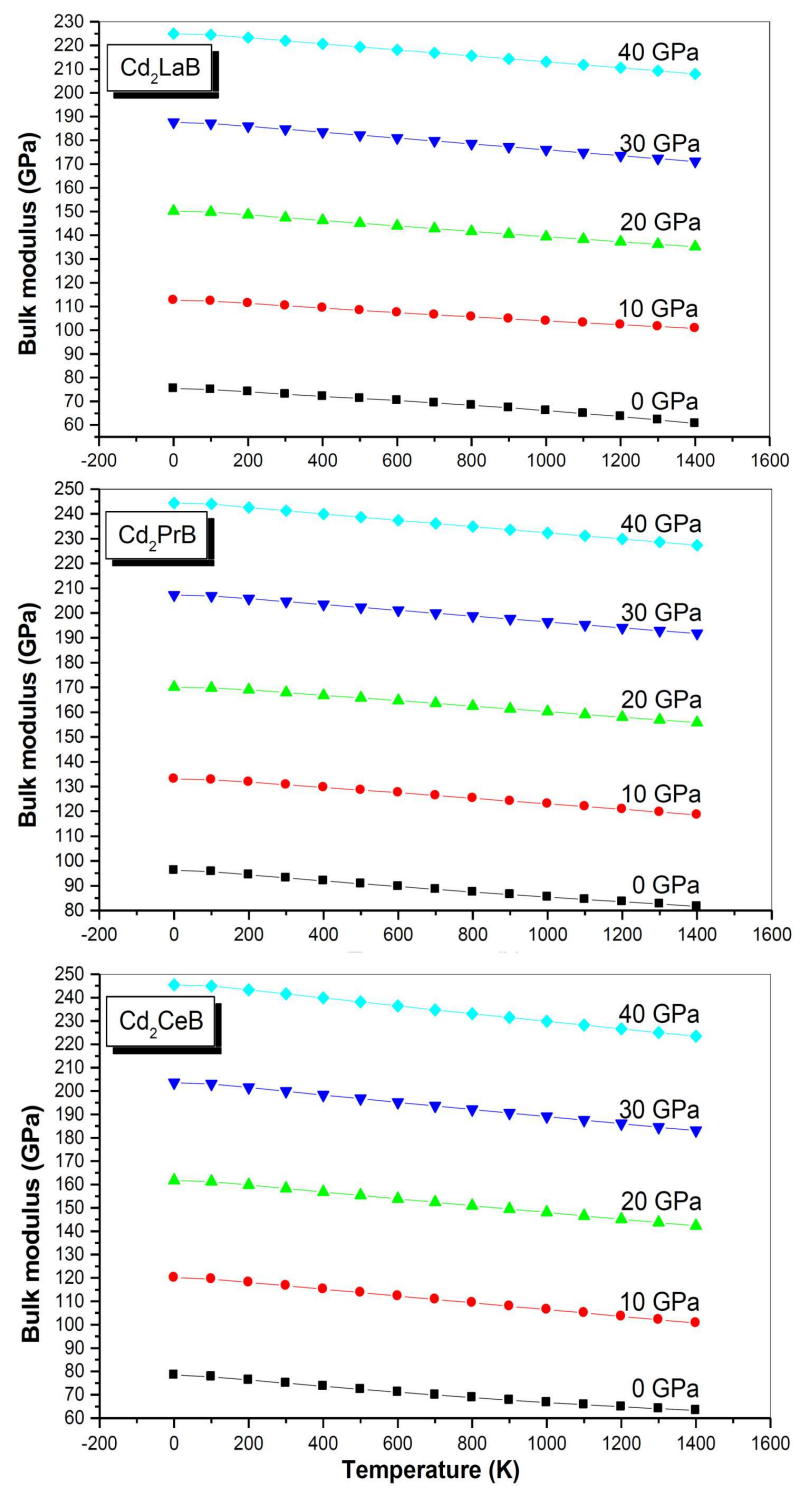

Fig. 8. The temperature effects on the bulk modulus of $\mathrm{Cd}_{2} \mathrm{LaB}, \mathrm{Cd}_{2} \mathrm{PrB}$, and $\mathrm{Cd}_{2} \mathrm{CeB}$ compounds.

These investigations demonstrate clearly that we can classify our compounds among the hard materials category due to their high Debye temperature. In fact, the Debye temperature of the full-Heusler alloys $\mathrm{Cd}_{2} \mathrm{LaB}$, $\mathrm{Cd}_{2} \mathrm{PrB}$, and $\mathrm{Cd}_{2} \mathrm{CeB}$ at equilibrium is 288.86, 321.17, and $288.93 \mathrm{~K}$, respectively.

In addition, relevant information about the lattice vibrations can be obtained by the constant volume heat capacity $C_{v}$. The investigation of $C_{v}$ as a function of the temperature at different pressures of $0,10,20,30$, and $40 \mathrm{GPa}$ is shown in Fig. 10. At high temperatures, $C_{v}$ tends to the Dulong and Petit limit, which is rather common for all solids [21]. At sufficiently low temperatures, $C_{v}$ is proportional to $T^{3}$ [22], while at equilibrium, the heat capacity for $\mathrm{Cd} 2 \mathrm{LaB}, \mathrm{Cd}_{2} \mathrm{PrB}$ and $\mathrm{Cd}_{2} \mathrm{CeB}$ compounds are found to be $98.967,97.873$, and 98.9118 $\mathrm{J} \mathrm{mol}^{-1} \mathrm{~K}^{-1}$, respectively.
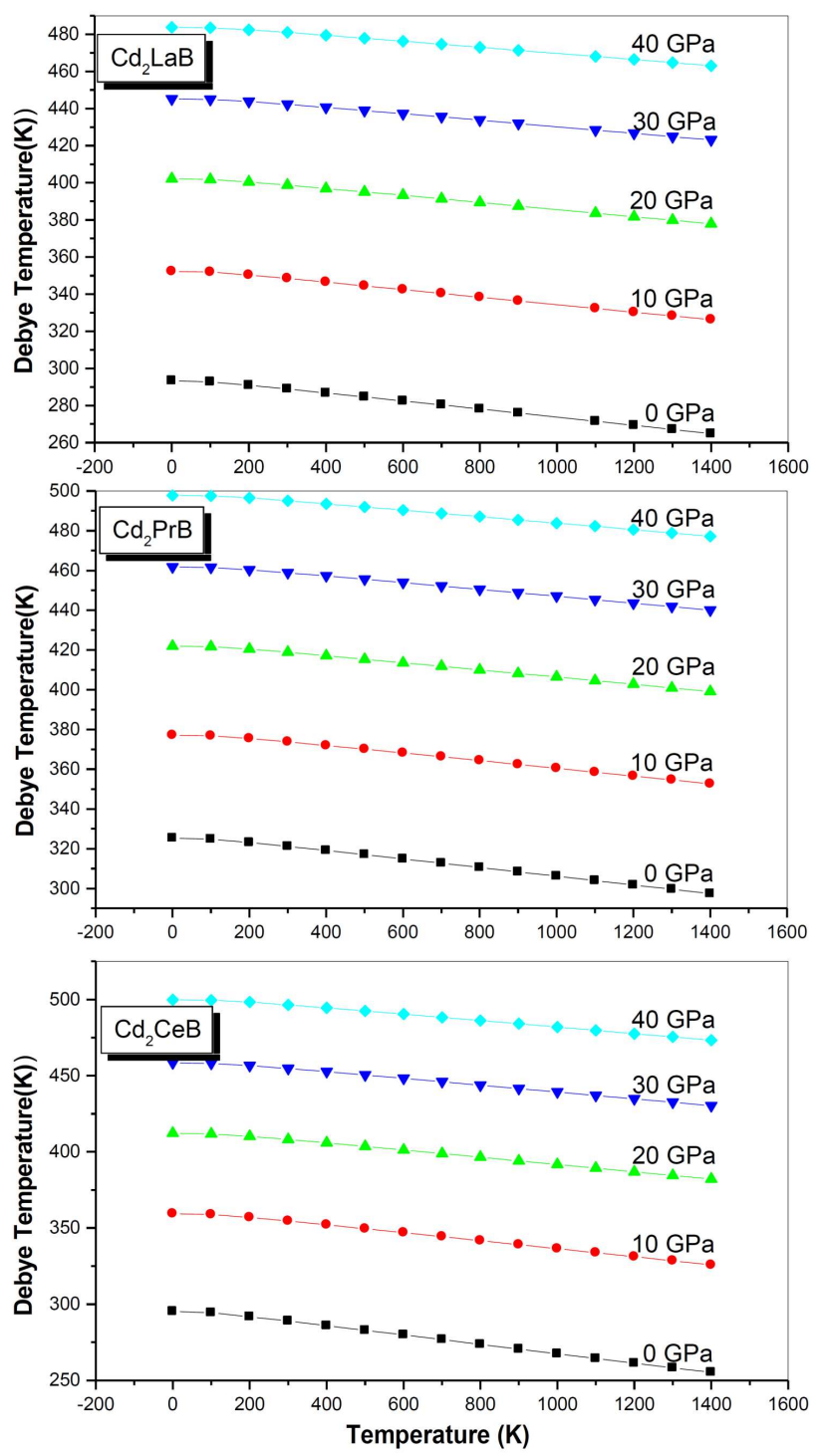

Fig. 9. Variation of the Debye temperature with respect to the temperature at different pressures ranging from 0 to $40 \mathrm{GPa}$ of $\mathrm{Cd}_{2} \mathrm{LaB}, \mathrm{Cd}_{2} \mathrm{PrB}$, and $\mathrm{Cd}_{2} \mathrm{CeB}$ compounds.

Figure 11 represents the variation of the thermal expansion coefficient $\alpha(T)$ of the $\mathrm{Cd}_{2} \mathrm{LaB}, \mathrm{Cd}_{2} \mathrm{PrB}$, and $\mathrm{Cd}_{2} \mathrm{CeB}$ as a function of the temperature and pressure. It is shown that, at a constant pressure and at low temperatures, $\alpha$ increases with temperature - especially at zero pressure - and gradually tends to a linear increase at higher temperatures. As the pressure increases, the increase of $\alpha$ with the temperature becomes smaller, while at constant temperature, $\alpha$ decreases strongly with increase of pressure.

The variation of the entropy $S$ as a function of temperature as illustrated by our results is displayed in Fig. 12 . It can be noticed that at fixed pressure $P$, entropy $S$ increases monotonously with the temperature $T$. The current investigations demonstrated that the lattice entropy 

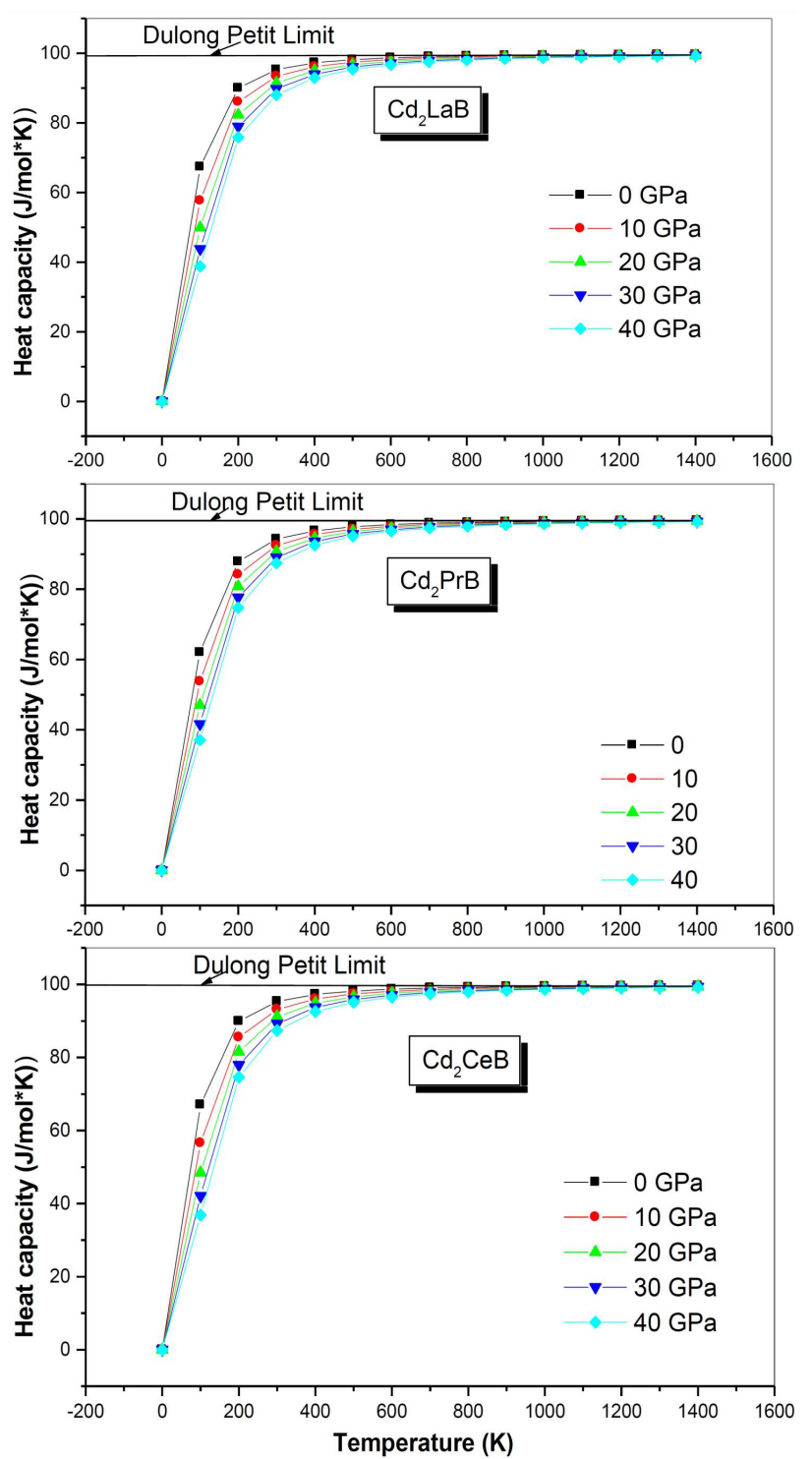

Fig. 10. The heat capacity depending on the temperature for different pressures from 0 to $40 \mathrm{GPa}$ of $\mathrm{Cd}_{2} \mathrm{LaB}$, $\mathrm{Cd}_{2} \mathrm{PrB}$, and $\mathrm{Cd}_{2} \mathrm{CeB}$ compounds.

shows strong pressure and temperature dependence. At $0 \mathrm{GPa}$ and $600 \mathrm{~K}, S=202 \mathrm{~J} \mathrm{~mol}^{-1} \mathrm{~K}^{-1}$. The thermal expansion is both of theoretical and practical importance and is essential for predicting the thermodynamic equation of state.

\section{Conclusions}

This work is a contribution to the study of the structural, elastic, magnetic, electronic, and thermodynamic properties of the $\mathrm{Cd}_{2} \mathrm{LaB}, \mathrm{Cd}_{2} \mathrm{PrB}$, and $\mathrm{Cd}_{2} \mathrm{CeB}$ using the FP-LMTO approach based on DFT within LDA and LSDA approximations. Our calculated network parameters and the bulk modulus are in good agreement with the theoretical data for similar materials. A numerical
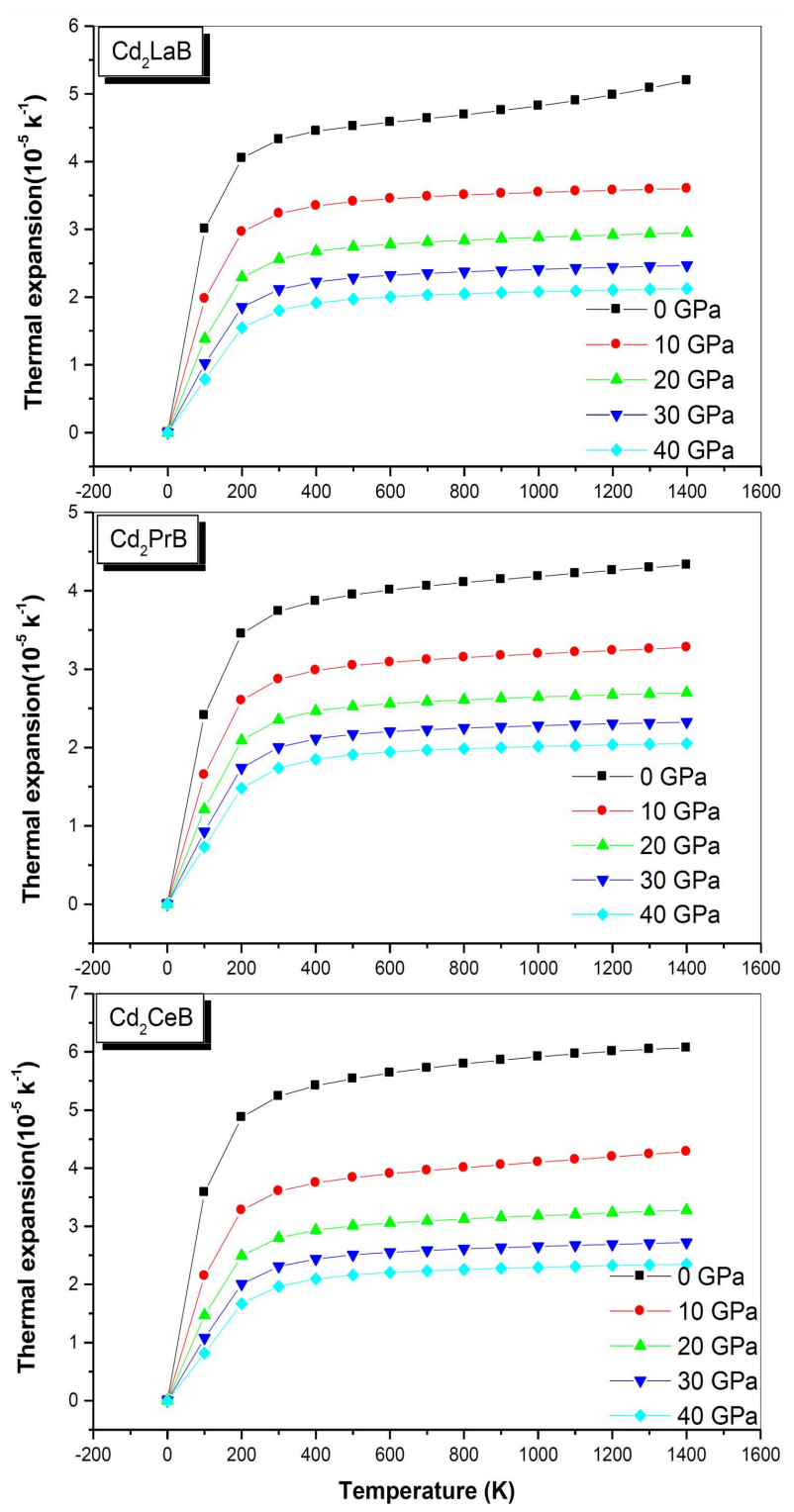

Fig. 11. The thermal expansion versus temperature and pressure.

first principles method was used to calculate the elastic constants $C_{11}, C_{12}$, and $C_{44}$. Our calculated total magnetic moment is very close to $3.334,3.482$, and $3.473 \mu_{\mathrm{B}}$ per unit cell.

The use of the quasi-harmonic Debye model was successfully applied to determine the thermal properties of $\mathrm{Cd}_{2} \mathrm{LaB}, \mathrm{Cd}_{2} \mathrm{PrB}$, and $\mathrm{Cd}_{2} \mathrm{CeB}$.

The theoretical heat capacity $C_{v}$ is close to the limit of Dulong-Petit, which is common to all high temperature solids. Since there are no experimental data available for these quantities, we believe that the theoretical estimate $a b$ initio is the only reasonable tool to obtain this important information and our calculated results also provide the reference for future experimental work. 

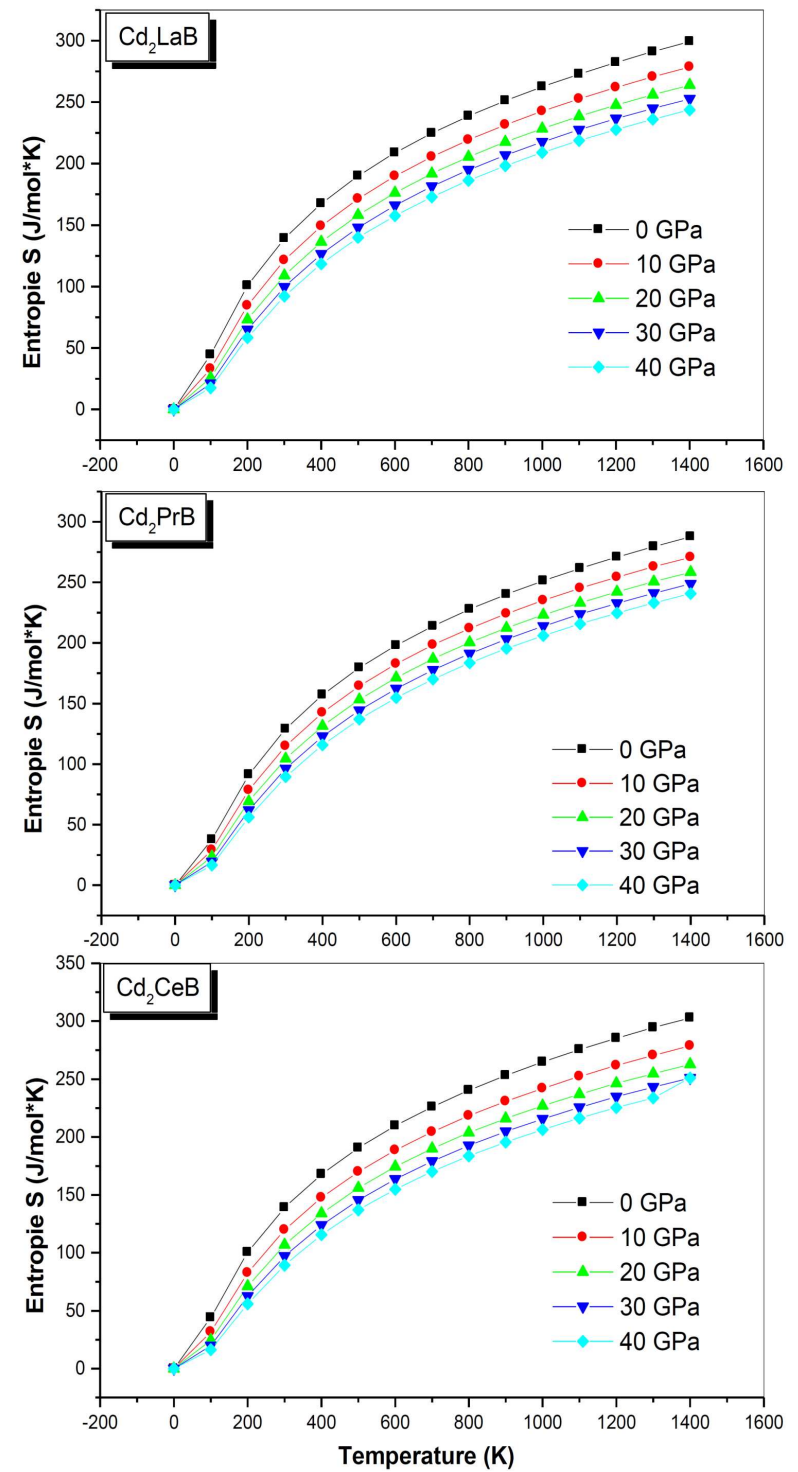

Fig. 12. The entropy versus temperature at pressures from 0 to $40 \mathrm{GPa}$ of $\mathrm{Cd}_{2} \mathrm{LaB}, \mathrm{Cd}_{2} \mathrm{PrB}$, and $\mathrm{Cd}_{2} \mathrm{CeB}$ compounds.

\section{References}

[1] P.J. Webster, Contemp. Phys. 10, 559 (1969).

[2] J. Kubler, A.R. Williams, C.B. Sommers, Phys. Rev. B 28, 1745 (1983).
[3] K.P.A. Ziebeck, P.J. Webster, J. Phys. Chem. Solids 25, 1 (1974).

[4] O. Heusler, Ann. Phys. 19, 155.22. (1934); P. Hohenberg, W. Kohn, Phys. Rev. 136, B864 (1964).

[5] J.P. Perdew, Y. Wang, Phys. Rev. B 45, 13244 (1992).

[6] J.P. Perdew, S. Burke, M. Ernwerhof, Phys. Rev. Lett. 77, 3865 (1996).

[7] J. Rath, A.J. Freeman, Phys. Rev. B 11, 2109 (1975).

[8] M.A. Blanco, A.M. Pendaś, E. Francisco, J.M. Recio, R. Franco, J. Mol. Struct. (Theochem.) 368, 245 (1996).

[9] M. Florez, J.M. Recio, E. Francisco, M.A. Blanco, A.M. Pendaś, Phys. Rev. B 66, 144112 (2002).

[10] E. Francisco, J.M. Recio, M.A. Blanco, A.M. Pendaś, J. Phys. Chem. 102, 1595 (1998).

[11] M.A. Blanco, E. Francisco, V. Luana, Comput. Phys. Commun. 158, 57 (2004).

[12] F.D. Murnaghan, Proc. Natl. Acad. Sci. USA 30 , 244 (1944).

[13] K.R.A. Ziebeck, P.J. Webster, J. Phys. Chem. Solids 35, 1 (1974).

[14] M.J. Mehl, J.E. Osburn, D.A. Papaconstantopoulos, B.M. Klein, Phys. Rev. B 41, 103112 (1990).

[15] M.J. Mehl, J.E. Osburn, D.A. Paraconstantantopoulos, B.M. Klein, Phys. Rev. B Condens. Matter 41, 10311 (1990).

[16] M. Ameri, F. Belkharroubi, B. Ameri, Y. A-Douri, B. Bouhafs, F.Z. Boufadi, A. Touia, K. Boudia, F. Mired, Mater. Sci. Semicond. Process. 26, 205 (2014).

[17] S. Benalia, M. Ameri, D. Rached, R. Khenata, M. Rabah, A. Bouhemadou, Comput. Mater. Sci. 43, 1022 (2008).

[18] M. Born, K. Huang, in: Dynamical Theory of Crystal Lattices, Oxford University Press, Clarendon 1956.

[19] M. Born, Proc. Cambridge Philos. Soc. 36, 160 (1940).

[20] M.A. Blanco, E. Francisco, V. Luoa, Comput. Phys. Commun. 158, 57 (2004).

[21] P. Debye, Ann. Phys. 39, 789 (1912).

[22] A.T. Petit, P.L. Dulong, Ann. Chim. Phys. 10, 395 (1819). 